\title{
A NEW METHOD OF HIP FUSION USING AN INTRAMEDULLARY NAIL
}

\section{A Preliminary Report}

\author{
Yutaka Onji, Yotaro Kurata and Hikaru Kido, Nara, Japan \\ From the Department of Orthopaedic Surgery, Nara Medical College, Kashiwara-shi, Nara-ken
}

With the improvement in the technique of arthroplasty and the increasing use of osteotomy fusion of the hip is indicated less frequently. When arthrodesis is required, good compensatory movement of the lumbar spine, correct position of the joint and full restoration of knee

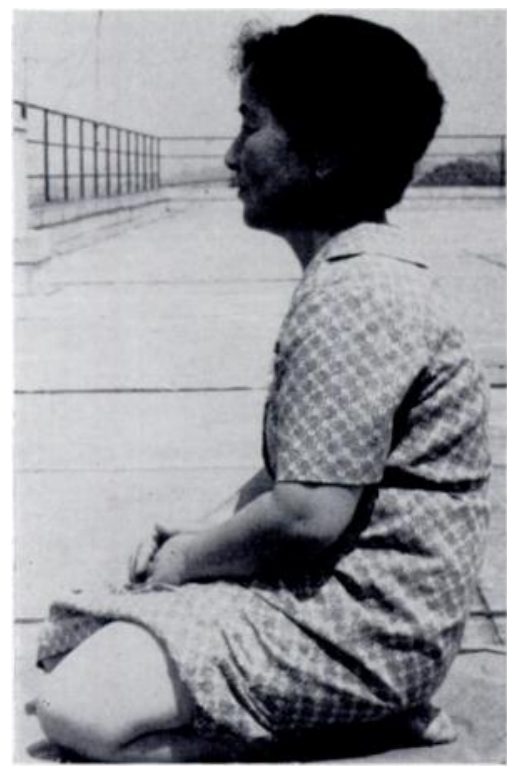

Fig. 1

A woman of 52 years who underwent fusion for osteoarthritis of the left hip in 1960, squatting in the Japanese movement must be ensured. Patients with a stiff hip can only squat when these requirements are met. Figure 1 shows a patient who had had her left hip fused in 1960 squatting in the Japanese way. Before 1958 we used to apply external fixation by plaster-of-Paris from the middle of the trunk down to the foot for about eight weeks. This led to stiffness of the spine and knee in almost all cases. When the duration of plaster fixation was more than a month a full range of knee movement was seldom obtained afterwards. To avoid the need for external fixation we have used intramedullary fixation since 1958 (Onji and Kurata 1958). A similar method of hip fusion was later reported by May and Mauck in 1963. The only difference is that we have used a straight nail rather than a slightly curved one.

\section{MATERIAL}

From March 1958 to August 1960 eighteen hips have been fused by this method in eighteen patients. The longest follow-up is seven years, and the shortest is over four years. The ages of the patients ranged from twelve to fifty-nine, and the proportion of men to women was equal. The conditions for which operation was performed were osteoarthritis in ten; fracture-dislocation of the hip in two; tuberculosis with fibrous union in five; and one old neglected congenital dislocation. Although the number of patients is small, we feel that the length of follow-up justifies this preliminary report.

\section{OPERATION}

The hip is approached either by the anterior Smith-Petersen incision or by a posterolateral one, but recently we have come to prefer the former. The acetabulum and femoral head are denuded to make direct contact between fresh bone surfaces. An intramedullary nail of correct length and diameter is chosen and a guide wire driven into the ilium through a small hole made in the antero-lateral quadrant of acetabular surface. The guide wire should come out through the highest point on the iliac crest. The intramedullary nail is then driven upwards into the ilium, after which the femur is reamed out to fit the nail and the upper end of the femur is placed in the acetabulum. The nail is then driven downwards into the femur. After the operation no external fixation is applied. Patients are allowed up with crutches from the tenth day after operation. Full weight bearing is allowed three months after operation and the nail is usually removed nine months later. 
The most important step in the whole procedure is to put the nail in the right place in the ilium. Figure 2 shows where this should be in the pelvis of a cadaver, with the nail entering the acetabulum through its antero-lateral quadrant, emerging from the ilium at its highest point, and traversing dense bone trabeculae.

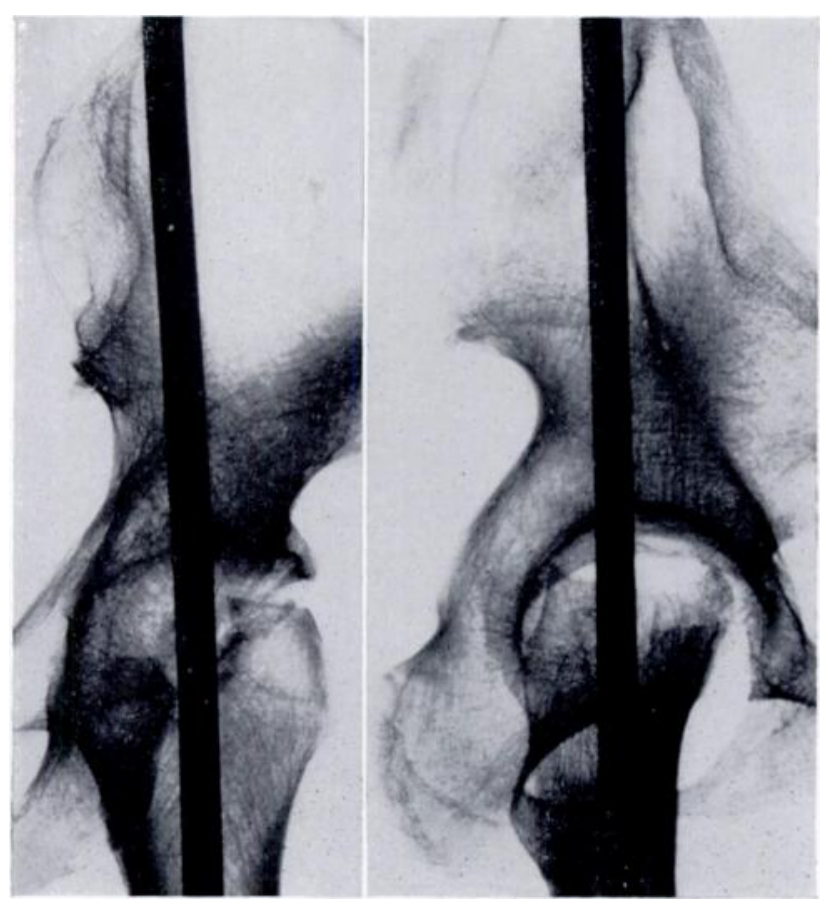

FIG. 2

Radiographs of a cadaveric pelvis showing the correct position of the nail.

\section{RESULTS}

In sixteen patients out of eighteen, fusion was prompt and complete. Figure 3 shows the patient with the longest follow-up period. He had a tuberculous hip with fibrous ankylosis and complained of pain and a limp on walking. Figure 4 shows the nail in position and Figure 5 shows complete bony fusion six and a half years later after removal of the nail. Figure 6 shows the hip of a patient with painful osteoarthritis. Figure 7 shows the appearance after operation and Figure 8 shows sound bony fusion four years later.

In one patient inadequate bony contact caused delayed union. Homogenous bone grafts were placed between the ilium and the trochanter and solid bony union was obtained. The reason for inadequate bone contact was that the femoral medullary cavity was not reamed out, so that when the nail was driven into the femur it pushed the femur downwards.

One patient developed a flare-up of a tuberculous infection which required drainage, and bony union was delayed.

No wound became infected. All the patients obtained a full range of knee movement and were able to squat in the Japanese way. No patient developed pain in the back or knee.

\section{DISCUSSION}

There are certain disadvantages in this technique. One is limitation of positions of the hip. As the nail has to go through the ilium, little choice is left in the angles of the hip both in the coronal and sagittal planes. In the sagittal plane the maximum angle of flexion is $\mathbf{3 0}$ degrees and in the coronal plane the maximum angle of abduction is 10 degrees. The other 


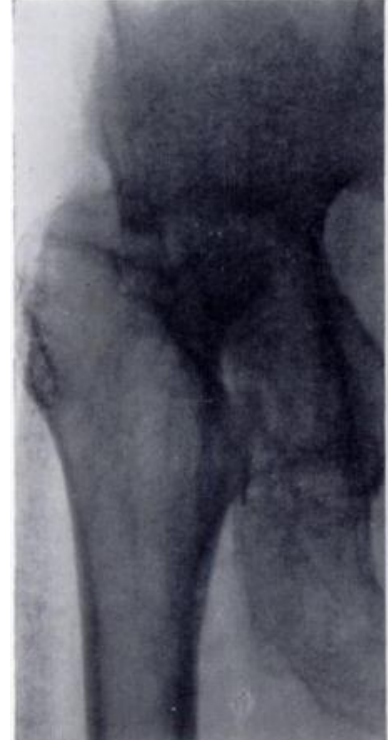

Fig. 3

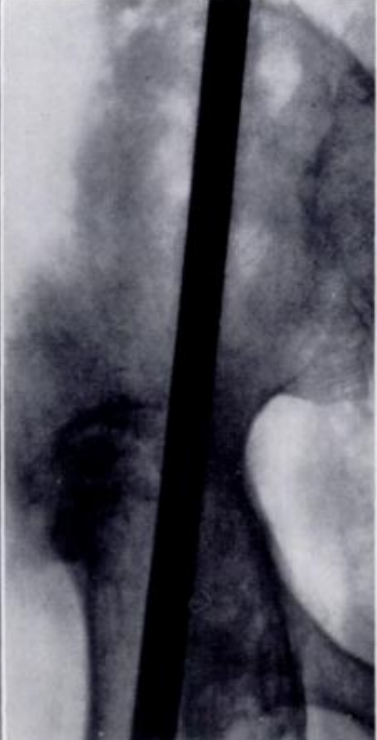

FIG. 4

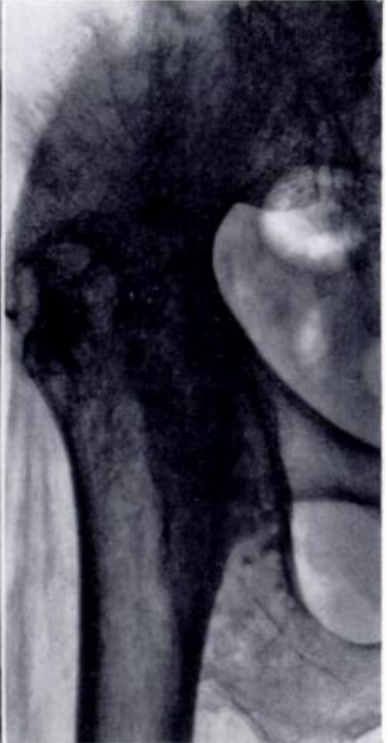

Fig. 5

Figure 3-Radiograph of a painful right hip due to old tuberculous infection in a man of 54 years. Figure 4-Radiograph after operation. Figure 5-Radiograph taken six and a half years later after removal of the nail, showing sound fusion.

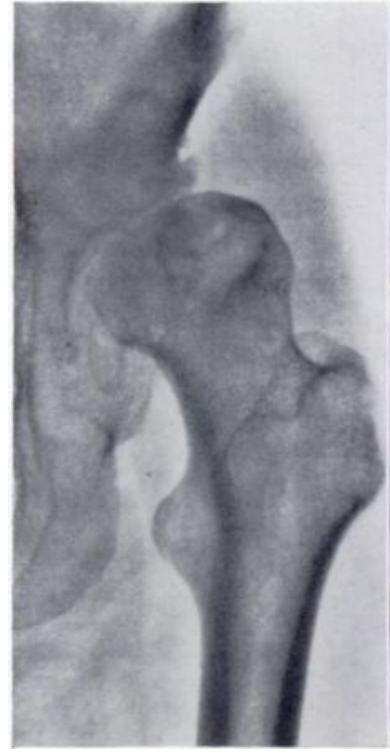

Fig. 6

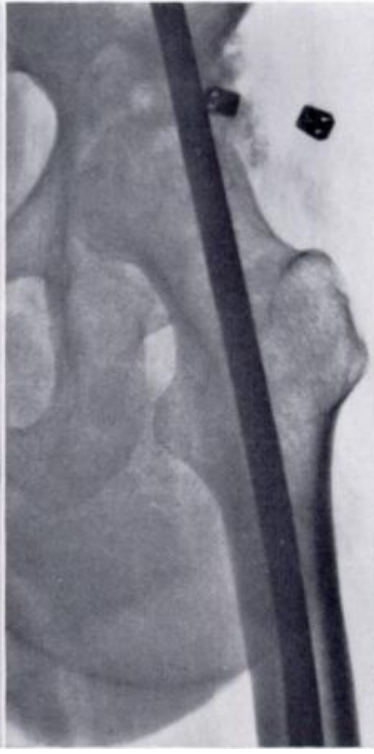

Fig. 7

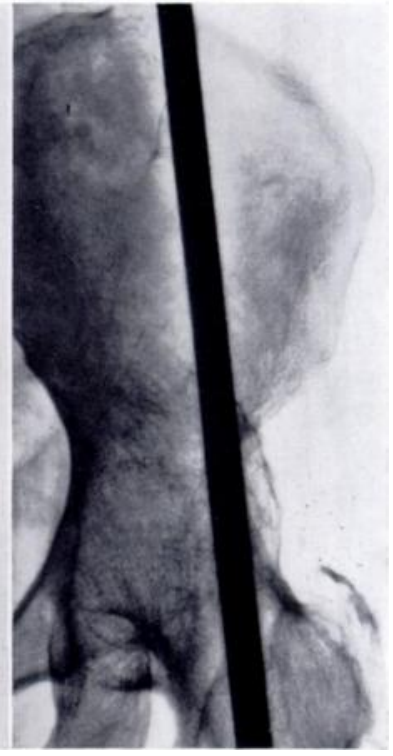

FiG. 8

Figure 6-Radiograph of the hip of the woman shown in Figure 1 with painful osteoarthritis. Figure 7-Showing the appearances after operation. Figure 8-Radiograph of the same patient four years later showing sound bony fusion. 
disadvantage is technical difficulty and extensive surgical exposure but, compared with the disadvantages of heavy external fixation by plaster-of-Paris, the technical difficulty is a small price to pay. Work is now under way to simplify the surgical technique and to reduce the extent of exposure.

As for the contra-indications of this technique, the patient with a flare-up of a tuberculous infection is the best example. If there is any risk of such an exacerbation this operation should not be done. As for special indications, old patients who cannot tolerate prolonged external fixation and bed rest can tolerate this method, and in a hot and humid climate where external fixation by plaster-of-Paris is most uncomfortable this method is the one of choice.

\section{SUMMARY}

1. A method of hip fusion with the aid of a straight intramedullary nail through the ilium and the femur is presented.

2. If properly done, no external fixation is necessary and the patient can be allowed up with crutches ten days after the operation.

3. The method has been used on eighteen hips between 1958 and 1960. There was one case of delayed union due to faulty technique and one case of exacerbation of an old tuberculous infection. Otherwise the follow-up study of the patients revealed good bony fusion in all cases, with no pain in the back or knee.

\section{REFERENCES}

MaY, V. R., Jun., and MaUCK, W. (1963): Intra-articular Hip Fusion with the Intramedullary Nail. Clinical Orthopaedics, 25, 113.

ONu, Y., and Kurata, Y. (1958): A New Surgical Technique. Central Japan Journal of Orthopaedic and Traumatic Surgery, 1, 282. 